\title{
Vers la souris transgénique drépanocytaire
}

Une équipe franco-américanocanadienne, dirigée par Yves Beuzard (Créteil, France), Marie Trudel (Montréal, Québec, Canada) et Frank Costantini (New York, NY, USA) vient de montrer que l'expression d'une super-hémoglobine $S$, l'hémoglobine $\mathrm{SAD}$ (Hb SAD), chez la souris transgénique induit un syndrome drépanocytaire. La souris transgénique SAD a été obtenue grâce à l'addition dans le gène $\beta^{\mathrm{s}}$ de deux mutations connues pour accroître la sévérité de la drépanocytose. Il s'agit de la mutation $\beta^{23}$ Ile (Antilles) et $\beta^{121}$ Glu (D. Punjab). Le gène $\beta^{\mathrm{SAD}}$, obtenu par mutagenèse dirigée, a été associé au LCR (locus control region) du locus des gènes de globine $\beta$. Rappelons que le LCR est une région de contrôle de tous les gènes $\beta$, située une quarantaine de $\mathrm{kb}$ en amont du gène $\beta$ adulte $\left(\mathrm{m} / \mathrm{s} n^{\circ} 6, \quad\right.$ p. 572 ; $n^{\circ} 7$, vol. 7, p. 740). Les constructions LCR $\beta^{\text {SAD }}$ et LCR gène $\alpha^{\text {humain }}$ ont été co-injectées dans l'œuf fécondé de souris. La lignée SAD-1 exprime $19 \%$ d'Hb SAD. Elle n'est pas malade à l'âge adulte, ni anémique, mais elle a un faible pourcentage de cellules anormales par leur petite taille, leur forme allongée et leur densité élevée. En outre, la polymérisation de l'hémoglobine et la falciformation surviennent lorsque les globules rouges SAD sont désoxygénés in vitro. Pour augmenter la sévérité de l'expression clinique de l'Hb SAD, la lignée SAD-1 a été croisée avec une lignée $\beta$-thalassémique (homozygote $\mathrm{Hbb}^{\mathrm{d} 3}$ ). Des souris issues de ce croisement sont composites, à la fois hétérozygotes $\beta$-thalassémiques $\left(\mathrm{Hb}^{\mathrm{th} /}\right)$ et hémizygotes SAD (souris $\beta$-thal SAD). Elles ont une proportion légèrement accrue d'Hb SAD (26 \%) provoquant des altérations de forme (forme allongée) et de densité des globules rouges semblables à celle des drépanocytes irréversibles (DI) humains. Les globules rouges des souris $\beta$-thal SAD-1 ont une durée de vie raccourcie comme le suggère une proportion élevée de réticulocytes $(6 \%$ au lieu de $\mathrm{m} / \mathrm{s} n^{\circ}$ 1, vol. 8, janviet 92
$2 \%$ ), une splénomégalie et une hyperplasie de l'érythropoïèse médullaire et splénique. Cette hémolyse anormale n'est pas due à la composante $\beta$ thalassémique (en elle-même sans conséquence à l'état hétérozygote) mais à la très forte capacité de polymérisation de l'hémoglobine $\mathrm{SAD}$, in vitro et in vivo. L'étude anatomopathologique révèle de nombreuses lésions histologiques caractéristiques de la drépanocytose dans différents organes, en particulier les reins, les poumons et la peau, la présence de drépanocytes irréversibles séquestrés contenant des fibres d'hémoglobine SAD polymérisée. L'hypoxie est fatale de manière spécifique chez $90 \%$ des souris $\beta$-thalSAD-1. Ainsi, la drépanocytose est patente chez la souris adulte, mais elle est moins sévère que chez l'homme, en particulier il n'y a pas d'anémie. En revanche, le syndrome drépanocytaire est très sévère au cours du développement précoce de la souris in utero, comme le montrent une mortalité très élevée avant sevrage (80 \%), l'hypotrophie du fotus et du placenta, le retard de croissance et l'anémie des nouveaunés survivants. Cette expression clinique très sévère de l'Hb $\mathrm{SAD}$ in utero peut être expliquée par la faible concentration de l'oxygène dans le sang fœtal, qui favorise la polymérisation de l'hémoglobine SAD du fotus, et par l'absence d'hémoglobine fotale chez la souris (qui, chez l'homme, est encore présente en quantité importante dans les premiers mois après la naissance). Ainsi, le syndrome drépanocytaire de la souris SAD-1 n'est pas assez sévère chez la souris adulte et trop sévère in utero pour être un modèle parfait de la maladie humaine. En attendant mieux, la souris $\beta$-thal SAD- 1 constitue cependant le premier modèle animal qui permet d'induire une crise vasoocclusive fatale par hypoxie et par conséquent d'étudier in vivo les agents antidrépanocytaires potentiels ayant une action sur la polymérisation de l'hémoglobine, la déshydratation du globule rouge et les mécanismes des obstructions vasculaires.

Les modèles précédemment créés de souris transgéniques expriment l'hémoglobine drépanocytaire humaine $\alpha_{2} \beta_{2}^{\mathrm{S}}$, dont $\mathrm{m} / \mathrm{s}$ s'est fait l'écho, ne présentaient, en effet, que peu ou pas d'anomalies du fait de la trop faible polymérisation de cette hémoglobine chez la souris. Il a donc fallu augmenter la polymérisation de l'hémoglobine $\mathrm{S}$, en ajoutant d'autres mutations existant également chez l'homme et augmentant la polymérisation de l'hémoglobine $\mathrm{S}$. La comparaison des propriétés des hémoglobines $\mathrm{S}$ et $\mathrm{SAD}$ semble cependant indiquer que les agent thérapeutiques potentiels actifs sur l'hémoglobine $\mathrm{S}$ et sur l'hémoglobine SAD devraient être les mêmes.

Y.B.

1. Trudel M, Saadane N, Garel MC, et al. Towards a transgenic mouse model of sickle cell disease : hemoglobin SAD. EMBO J $1991 ; 10$ : 3157-65.

\section{AVIS AUX AUTEURS DE TRAVAUX IMPORTANTS}

$\mathrm{m} / \mathrm{s}$ propose aux auteurs de travaux importants, publiés dans des revues d'audience internationale et de premier niveau, de présenter leurs résultats sous forme de brève, de nouvelle, voire de mini-synthèse, au mieux publiés dans médecine/sciences parallèlement à l'article princeps.

\section{LA RÉDACTION}

Les manuscrits doivent être adressés à : médecine/sciences, 6, ne Blanche, 92120 Montrouge, France.

Tel. : (1) 47.35 .85 .52

Fax : 46.57 .10 .09 